\title{
The evolution of damped Ly- $\alpha$ absorbers: metallicities and star formation rates
}

\author{
Varsha P. Kulkarni ${ }^{1}$, Donald G. York ${ }^{2}$, James T. Lauroesch ${ }^{3}$, \\ S. Michael Fall ${ }^{4}$, Pushpa Khare ${ }^{5}$, Bruce E. Woodgate ${ }^{6}$, \\ Povilas Palunas $^{7}$, Joseph Meiring ${ }^{1}$, Deepashri G. Thatte ${ }^{1}$, \\ Daniel E. Welty ${ }^{2}$ and James W. Truran ${ }^{2}$ \\ ${ }^{1}$ Dept. of Physics and Astronomy, Univ. of South Carolina, Columbia, SC 29208, USA \\ emails: kulkarni@sc.edu; meiring@physics.sc.edu; thatte@physics.sc.edu \\ ${ }^{2}$ Dept. of Astronomy and Astrophysics, Univ. of Chicago, Chicago, IL 60637, U.S.A. \\ email:don@oddjob.uchicago.edu \\ ${ }^{3}$ Dept. of Physics and Astronomy, Northwestern Univ., Evanston, IL 60208, USA \\ email: jtl@elvis.astro.northwestern.edu \\ ${ }^{4}$ Space Telescope Science Inst., Baltimore, MD 21218, U.S.A. \\ email:fall@stsci.edu \\ ${ }^{5}$ Dept. of Physics, Utkal University, Bhubaneswar, 751004, India \\ email:khare@iopb.res.in \\ ${ }^{6}$ NASA/Goddard Space Flight Center, Code 681, Greenbelt, MD 20771, U.S.A. \\ email:woodgate@uit.gsfc.nasa.gov \\ ${ }^{7}$ McDonald Observatory, Univ. of Texas, \\ Austin, TX 78712, U.S.A \\ email: palunas@astro.as.utexas.edu
}

\begin{abstract}
Damped Lyman- $\alpha$ (DLA) and sub-DLA quasar absorption lines provide powerful probes of the evolution of metals, gas, and stars in galaxies. One major obstacle in trying to understand the evolution of DLAs and sub-DLAs has been the small number of metallicity measurements at $z<1.5$, an epoch spanning $\sim 70 \%$ of the cosmic history. In recent surveys with the Hubble Space Telescope and Multiple Mirror Telescope, we have doubled the DLA Zn sample at $z<1.5$. Combining our results with those at higher redshifts from the literature, we find that the global mean metallicity of DLAs does not rise to the Solar value at low redshifts. These surprising results appear to contradict the near-Solar mean metallicity observed for nearby $(z \approx 0)$ galaxies and the predictions of cosmic chemical evolution models based on the global star formation history. Finally, we discuss direct constraints on the star formation rates (SFRs) in the absorber galaxies from our deep Fabry-Perot Ly- $\alpha$ imaging study and other emission-line studies in the literature. A large fraction of the observed heavy-element quasar absorbers at $0<z<3.4$ appear to have SFRs substantially below the global mean SFR, consistent with the low metallicities observed in the spectroscopic studies.
\end{abstract}

\section{Introduction}

Heavy-element quasar absorption systems probe galaxies at various epochs, selected independent of their luminosities. The damped Ly-alpha absorbers (DLAs; $\log N_{\mathrm{HI}}>$ 20.3) and sub-DLAs $\left(19.0<\log N_{\mathrm{HI}}<20.3\right)$ constitute a large fraction of $\mathrm{H} \mathrm{I}$ in galaxies (e.g. Wolfe et al. 1995; Peroux et al. 2003), and provide the best existing probes of the chemical composition of galaxies over $\sim 90 \%$ of the cosmic history. They should thus provide important clues to the history of metal production and star formation in galaxies. 


\section{Evolution of metallicity}

Most cosmic chemical evolution models predict the global mean interstellar metallicity of galaxies to rise with time, from low values at high redshifts to Solar or near-Solar values at $z=0$ (e.g. Pei \& Fall 1995; Malaney \& Chaboyer 1996; Pei, Fall \& Hauser 1999; Somerville et al. 2001). This rise in metallicity at low redshifts is driven by the high global star formation rate (SFR) at $z \gtrsim 1.5$ implied by galaxy imaging surveys such as the Canada-France Redshift Survey (CFRS) and the Hubble Deep Field (HDF). (see e.g. Lilly et al. 1996; Madau et al. 1996). Indeed, the mass-weighted mean metallicity of nearby galaxies is also near-Solar (e.g. Kulkarni \& Fall 2002). Do the DLA data show the predicted rise in the global mean metallicity with decreasing redshift?

We adopt $\mathrm{Zn}$ as our primary metallicity indicator because (a) Zn tracks Fe closely, to within $\sim \pm 0.1$ dex in the Galactic halo and disk stars for $[\mathrm{Fe} / \mathrm{H}] \gtrsim-3$; (b) $\mathrm{Zn}$ is relatively undepleted on interstellar dust grains; and (c) the Zn II $\lambda \lambda 2026,2062$ lines in DLAs often lie outside the Lyman- $\alpha$ forest and are usually unsaturated. In the absence of selection effects, the quantity $\Omega_{\text {metals }}^{\mathrm{ISM}} / \Omega_{\text {gas }}^{\mathrm{ISM}}$ is equal to the $N_{\mathrm{HI}}$-weighted mean metallicity $\bar{Z}$ in a sample of DLAs, where $\bar{Z} / Z_{\odot}=\left[\Sigma N(\mathrm{Zn} \mathrm{II})_{i} / \Sigma N(\mathrm{HI})_{i}\right] /(\mathrm{Zn} / \mathrm{H}) \odot($ e.g. Lanzetta et al. 1995; Kulkarni \& Fall 2002). There has been considerable debate about whether or not this quantity $\bar{Z}$ rises with decreasing redshift. Based on $57 \mathrm{Zn}$ measurements at $0.4<z<3.4$, Kulkarni \& Fall (2002) found the slope of the metallicity-redshift relation to be $-0.26 \pm 0.10$, consistent at the $\approx 2-3 \sigma$ level with both the predicted rates of evolution $(-0.25$ to -0.61$)$, and with no evolution. Prochaska et al. (2003) reached similar conclusions (slope $-0.25 \pm 0.07$ ) for $0.5<z<4.7$, combining $\mathrm{Zn}$, Fe, Si, S, O, and $\mathrm{X}$-ray absorption measurements in 121 DLAs. The main reason for the debate about this issue is the small number of measurements available, especially at $z<1.5$. Space-based ultraviolet (UV) measurements are needed to access the H I Ly- $\alpha$ lines at $z<1.6$ and the Zn II lines at $z<0.6$. Furthermore, for $0.6<z<1.5$, the Zn II lines lie in the blue region, where most spectrographs have relatively low sensitivity. To improve this situation, we have recently started to expand the DLA abundance samples at $z<1.5$, using the Multiple Mirror Telescope (MMT) and the Hubble Space Telescope (HST).

\subsection{Abundance measurements for DLAs at $z<1.5$}

The low- $z$ end is important to clarify the overall shape of the metallicity-redshift relation and to understand the relation of DLAs to present-day galaxies. To constrain the low- $z$ end better, we carried out HST STIS observations of 4 DLAs with $0.09<z<0.52$ and $20.3<\log N_{\mathrm{HI}}<21.3$ (Kulkarni et al. 2005a). 5-10 orbits were spent per object with the STIS G230M/NUV-MAMA or G230MB/CCD configurations at spectral resolutions of 10,400-14,300. The data were reduced using the IRAF and STSDAS/CALSTIS packages. Column densities were estimated by fitting the line profiles and verified using the apparent optical depth method. Three of the four DLAs in our sample, where we could put meaningful constraints on the $\mathrm{Zn}$ abundance, have $\mathrm{Zn} / \mathrm{H}$ below 10-20\% Solar.

To expand the DLA Zn samples in the intermediate-redshift range, we have been carrying out a spectroscopic survey of DLAs with $0.6<z<1.5$ using the MMT blue channel spectrograph. Despite the modest resolution $\left(\sim 75 \mathrm{~km} \mathrm{~s}^{-1}\right)$ of this instrument, we have been able to detect lines of $\mathrm{Zn}, \mathrm{Cr}, \mathrm{Fe}, \mathrm{Mn}, \mathrm{Ni}$, Ti, etc. because of the high $\mathrm{S} / \mathrm{N}$ in our spectra. Some of the data from this ongoing survey are described by Khare et al. (2004). The Zn abundances for the DLAs in this sample were found to be 3-32 \% Solar.

\subsection{Implications for the global metallicity-redshift relation}

Our HST and MMT data have so far doubled the DLA Zn sample at $z<1.5$ and tripled the sample at $z<1$. Fig. 1 shows the logarithm of the $N_{\mathrm{HI}}$-weighted mean $\mathrm{Zn}$ 

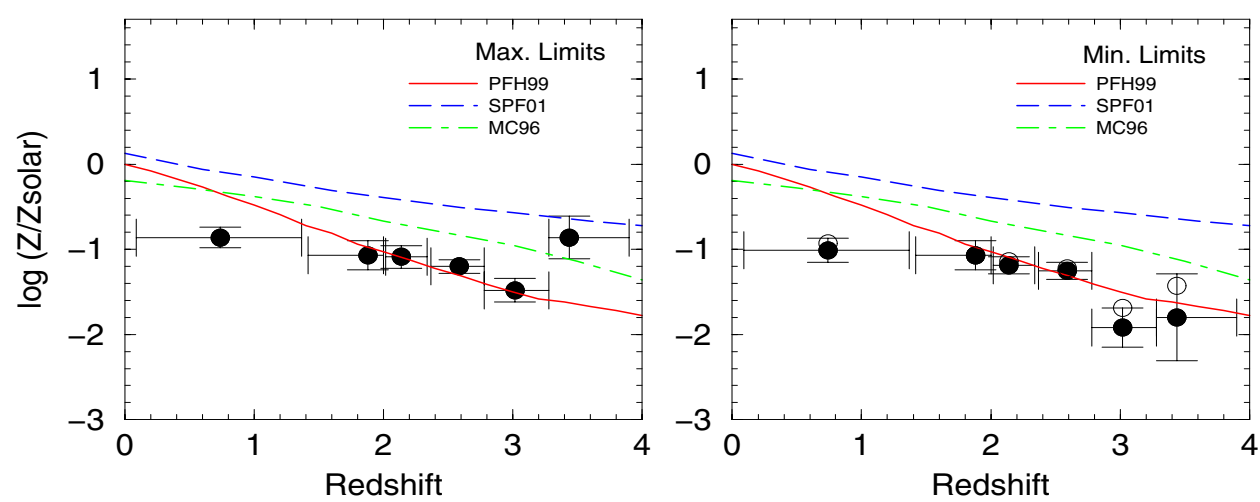

Figure 1. Global metallicity-redshift relation for DLAs from 87 measurements, based on our HST and MMT data and the literature. The circles show the logarithm of the $N(\mathrm{HI})$-weighted mean Zn metallicity relative to the Solar value. The filled circles in the left and right panels show the results with the Zn upper limits treated as detections and zeros respectively. The unfilled circles in the right panel show the results using information from other elements in those cases where only limits rather than detections are available for Zn. The solid, short-dashed, and dot-dashed curves show respectively the "true" mean metallicity (not corrected for dust obscuration) expected in the cosmic chemical evolution models of Pei et al. (1999), Somerville et al. (2001), and Malaney \& Chaboyer (1996).

metallicity as a function of redshift, for the sample of 87 DLAs, based on our HST and MMT results so far and the values available in the literature (see Kulkarni et al. 2005a and references therein). Horizontal bars for each bin show the range of DLA redshifts within that bin. The vertical error bars show the $1 \sigma$ uncertainties in the $N_{\mathrm{HI}}$-weighted mean metallicities, and include both the measurement uncertainty and the sampling uncertainty. The left panel shows the "maximum-limits" case, where the Zn upper limits are treated as detections. The filled circles in the right panel show the "minimum-limits" case with the $\mathrm{Zn}$ limits treated as zeros. The unfilled circles in the right panel show the modified minimum-limits sample, using other elements to constrain the metallicities in cases of $\mathrm{Zn}$ limits. The slopes of the metallicity-redshift relation in the range $0.09<$ $z<3.90$ for these three cases are $-0.18 \pm 0.06,-0.22 \pm 0.08$, and $-0.23 \pm 0.06$. The corresponding estimates of the $z=0$ intercept of the metallicity-redshift relation are $-0.74 \pm 0.15,-0.75 \pm 0.18$, and $-0.71 \pm 0.13$ respectively. Clearly, the global mean metallicity of DLAs does not seem to rise up to the Solar value at low redshifts, and shows at best a slow evolution at a rate of $\approx 0.2$ dex or less per unit redshift. This result appears to contradict the predictions from most cosmic chemical evolution models and the global star formation history. We return to the implications of this in section 4 .

\section{Evolution of star formation rates}

Another important quantity necessary to study the evolution of an absorber galaxy is its SFR. The SFR can be estimated from emission lines such as Ly- $\alpha, \mathrm{H}-\alpha$, [O II], or [O III] seen commonly in star-forming regions. Searches for low- $z$ DLAs have often imaged, and sometimes spectroscopically confirmed the absorbing galaxies (e.g. Yanny et al. 1990; Le Brun et al. 1997; Chen et al. 2005). However, this has been much more difficult at high redshifts. There have been a few detections of Ly- $\alpha$ emission in high$z$ quasar absorber fields (e.g. Lowenthal et al. 1991; Francis et al. 1996; Roche et al. 2000). However, most other attempts to detect Ly- $\alpha$ emission from high- $z$ intervening 

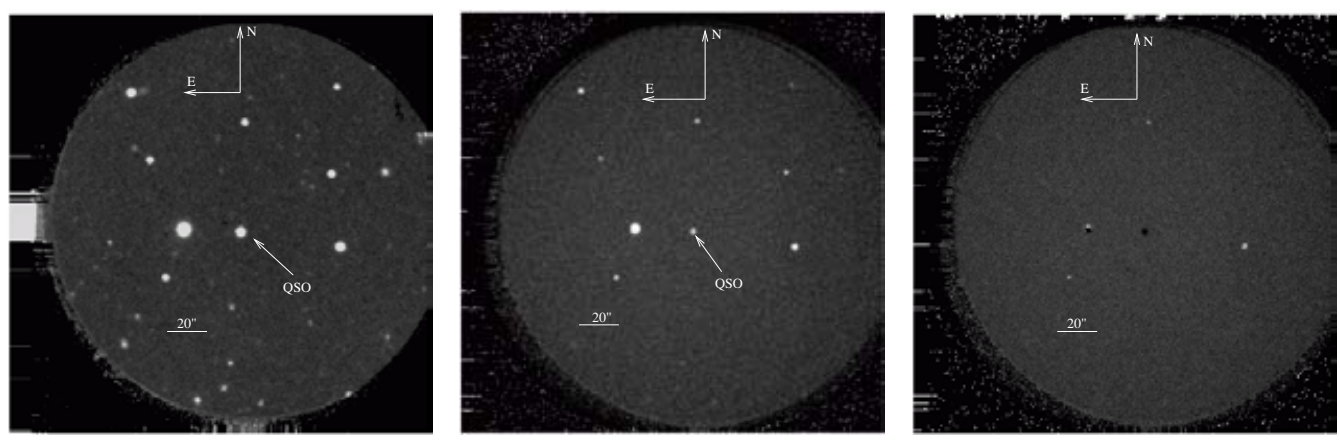

Figure 2. APO images of the field of Q0216+080 in B band (left) and in narrow-band centred on Ly- $\alpha$ emission at $z=2.2931$ before (centre) and after (right) continuum subtraction.

$\left(z_{a b s}<z_{e m}\right)$ DLAs have produced either non-detections or weak detections. Most of the few confirmed Ly- $\alpha$ detections in high-redshift DLAs have been for absorbers with $z_{a b s} \approx z_{e m}$, which may differ from the cosmologically more interesting general population of DLAs with $z_{a b s}<z_{e m}$. Several attempts to detect DLAs in H- $\alpha$ have either yielded non-detections or detected companions separated by large angular distances from the quasars, rather than objects close to quasar sight-lines.

We have been carrying out a narrow-band Fabry-Perot (FP) imaging survey of quasar absorber fields at the Apache Point Observatory 3.5 meter telescope (Kulkarni et al. 2005b). The blue and vis-broad etalons in the Goddard Space Flight Center (GSFC) Fabry Perot (FP) imaging system have optimum sensitivity and resolution in the wavelength range $\sim 4000-5000 \AA$. We therefore restricted our search to the redshift range $2.3<z<3.1$. We searched the York et al. (1991) catalogue of heavy-element quasar absorbers for absorbers with (i) $2.3<z_{a b s}<3.1$, (ii) $z_{a b s}<z_{e m}-0.6$ to avoid absorbers possibly associated with the quasars, and (iii) with well-detected mixed-ionisation lines (Si II, Al II, or O I in addition to C IV and/or Si IV). Six such absorbers, including 2 DLAs, were finally observed.

The observations were carried out during 9 runs between October 2000 and May 2004 at the 3.5 meter Apache Point Observatory (APO) telescope. Total integration times were 320-600 minutes per field, making these among the deepest images ever taken for quasar absorber fields. Data reduction was carried out using standard IRAF tasks. Figs. $2 \mathrm{a}$ and $2 \mathrm{~b}$ show the reduced broad-band and narrow-band images of one of our fields, Q0216+080, which is known to have a DLA at $z=2.2931$. The stripes at the borders of some images are an artifact of the co-adding of the dithered images. The quasar is considerably dimmer in the narrow-band image because of the presence of the foreground DLA. Fig. 2c shows the continuum-subtracted image, obtained by aligning the images spatially, and adjusting the scaling factor so as to minimise variance in the central portion of the subtracted image. All of the objects in the narrow-band images disappeared almost completely after subtraction of the continuum. The slight residuals left at the positions of some stars are because of the difficulty in matching the point spread functions (PSFs) perfectly in the broad-band and narrow-band images. No significant Ly- $\alpha$ emission was detected from any object in this or any of the other quasar absorber fields in our sample. To estimate the limits on the Ly- $\alpha$ fluxes for the absorbers in these fields, we used our observations of the field of the radio galaxy 53w002 with known Ly- $\alpha$ emitters at $z=2.39$ (Pascarelle et al. 1996), and observations of a standard star. Based on this, the $3 \sigma$ observed-frame Ly- $\alpha$ flux sensitivity reached in our images is in the range of $1.9-5.4 \times 10^{-17} \mathrm{erg} \mathrm{s}^{-1} \mathrm{~cm}^{-2}$, implying $3 \sigma$ SFR limits of $0.8-2.4 \mathrm{M}_{\odot} \mathrm{yr}^{-1}$. 


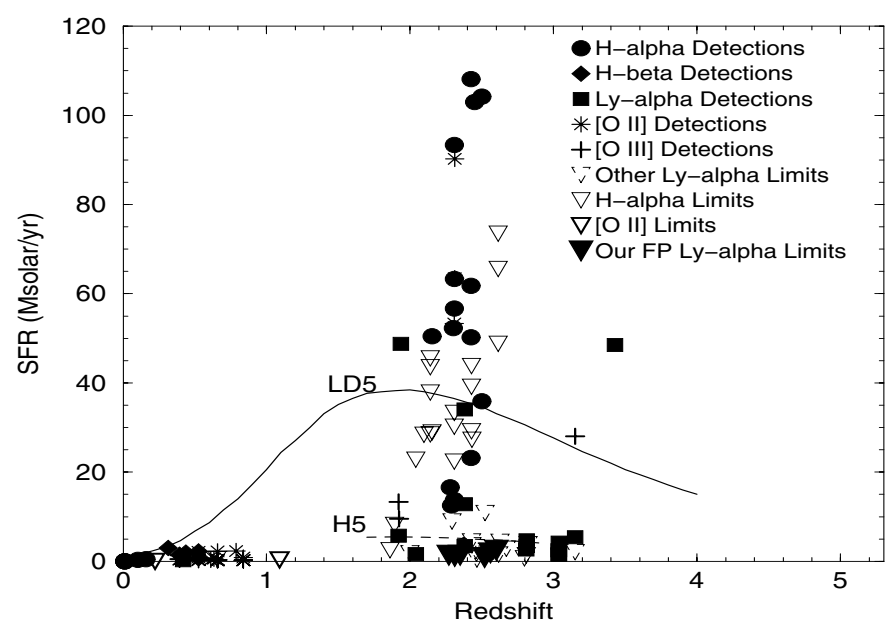

Figure 3. Measurements of star formation rates (in $M_{\odot} \mathrm{yr}^{-1}$ ) for confirmed or candidate objects in quasar absorber fields, from emission line searches. Data points are from our APO FP survey, our previous NICMOS imaging (Kulkarni et al. 2000, 2001), and other literature (Kulkarni et al. 2005b, and references therein). The curves show the calculations of Bunker et al. (1999) for the predicted cross-section-weighted SFR in the large-disk and hierarchical scenarios.

The lack of Ly- $\alpha$ emitters in our fields, while surprising, does not contradict the space density of Ly- $\alpha$ emitters seen in other studies at similar redshifts (e.g. Stiavelli et al. 2001; Palunas et al. 2004). Both these studies found several LAEs, but were much larger in field of view and redshift depth than our study. The field of Stiavelli et al. was a non-absorber field. The field of Palunas et al. does show some sub-DLAs, but is known to be a filament with higher density than a typical field region.

Fig. 3 plots our APO FP SFR limits with our earlier NICMOS results at $z \sim 1.9$ (Kulkarni et al. 2000, 2001), and the results of other emission line searches in quasar absorbers (see references in Kulkarni et al. 2005b). All data points have been converted to a common cosmological model with $\Omega_{m}=0.3, \Omega_{\Lambda}=0.7$, and $H_{0}=70 \mathrm{~km} \mathrm{~s}^{-1} \mathrm{Mpc}^{-1}$. The majority of the data in Fig. 3 are for DLAs. We note, however, that many of the $\mathrm{H}-\alpha$ and Ly- $\alpha$ candidates have not yet been spectroscopically confirmed. Furthermore, many of the $\mathrm{H}-\alpha$ points are for objects at large angular separations from the quasar. Our APO FP limits are among the tightest existing constraints on SFRs in absorption-selected galaxies, but are clearly consistent with many other measurements. The solid curve shows the LD5 calculation of Bunker et al. for the predicted cross-section-weighted SFR based on the closed-box Pei \& Fall (1995) model, for large proto-spirals (with space density equal to that of local spirals) for $q_{0}=0.5$ and $H_{0}=70 \mathrm{~km} \mathrm{~s}^{-1} \mathrm{Mpc}^{-1}$. The dashed curve shows their H5 prediction for the hierarchical hypothesis (with a higher absorber space density in the past). Clearly, a large fraction of the observed SFR values fall below the prediction of the large-disk scenario, and several lie even below the hierarchical prediction. Even though the predicted SFRs for $\Omega_{m}=0.3$ and $\Omega_{\Lambda}=0.7$ would be somewhat lower, a large fraction of the absorbers would still have SFRs below the LD5 predictions.

\section{Conclusions}

Our results on both metallicities and SFRs appear to suggest that the history of metal production and star formation may have been quite different in DLAs than in the general 
galaxy population. Is this an effect of small number statistics? Or is this a selection effect caused by the fact that the more metal-rich and more vigorously star-forming DLAs are also likely to be dustier and may thus be under-represented in flux-limited samples of quasars? Or are the star-forming regions in DLAs compact and at small angular separations from the quasars, so that they get lost in the quasar point spread function (PSF)? Clearly, it is necessary to expand the DLA metallicity samples at $z<1.5$ to better understand the metallicity evolution. Such studies, together with high-resolution optical/IR imaging and spectroscopic confirmation of the absorbing galaxies will help to understand the overall role of DLAs in the big picture of galaxy evolution.

\section{Acknowledgements}

We thank the organisers for holding this very stimulating conference. VPK, SMF, and JTL acknowledge partial support from the NASA/STScI grant GO-9441. VPK also acknowledges partial support from the U. S. National Science Foundation grant AST0206197 and the Univ. of South Carolina Research Foundation. DEW acknowledges support from the NASA LTSA grant NAG5-11413. JWT acknowledges support from the NSF Physics Frontier Center, Joint Institute for Nuclear Astrophysics under grant PHY 02-16783 and DOE support under grant DE-FG 02-91ER 40606.

\section{References}

Bunker A. J., et al., 1999, MNRAS, 309, 875

Chen, H.-W., Kennicutt, R. C., Rauch, M. 2005, ApJ, 620, 703

Fall, S. M., Pei, Y. C., 1993, ApJ, 402, 479

Francis, P. J., et al., 1996, ApJ, 457, 490

Khare, P., Kulkarni, V., Lauroesch, J., York, D., Crotts, A., Nakamura, O. , 2004, ApJ, 616, 86

Kulkarni, V. P., et al., 2000, ApJ, 536, 36

Kulkarni, V. P., et al., 2001, ApJ, 551, 37

Kulkarni, V. P., Fall, S. M., 2002, ApJ, 580, 732

Kulkarni, V. P., Fall, S. M., Lauroesch, J. T., York, D. G., Welty, D. E., Khare, P., Truran, J. W., 2005a, ApJ, 618, 68

Kulkarni, V. P., Woodgate, B. E., York, D. G., Thatte, D. G., Meiring, J., Palunas, P., Wassell, E., 2005b, ApJ, submitted

Le Brun, V., Bergeron, J., Boisse, P., Deharveng, J.-M., 1997, A\&A, 321, 733

Lilly, S. J., Le Fevre, O., Hammer, F., Crampton, D., 1996, ApJ, 460, L1

Lowenthal, J. D., et al., 1991, ApJ, 377, L73

Madau, P., et al., 1996, MNRAS, 283, 1388

Malaney, R. A., Chaboyer, B., 1996, ApJ, 462, 57

Palunas, P., et al., 2004, ApJ, 602, 545

Pascarelle, S. M., et al., 1996, ApJ, 456, L21

Pei, Y. C., Fall, S. M., 1995, ApJ, 454, 69

Pei, Y. C., Fall, S. M., Hauser, M. G., 1999, ApJ, 522, 604

Péroux, C., et al., 2003, MNRAS, 345, 480

Prochaska, J. X., Gawiser, E., Wolfe, A. M., Castro, S., Djorgovski, S. G., 2003, ApJ, 595, L9

Roche, N., Lowenthal, J. Woodgate, B., 2000, ApJ, 317, 937

Somerville, R. S., Primack, J. R., Faber, S. M., 2001, MNRAS, 320, 504

Stiavelli, M., Scarlata, C., Panagia, N., Treu, T., Bertin, G., Bertola, F., 2001, ApJ, 561, L37

Wolfe, A. M., Lanzetta, K. M., Foltz, C. B., Chaffee, F. H., 1995, ApJ, 454, 698

Yanny, B., York, D. G., Williams, T. B., 1990, ApJ, 351, 377

York, D. G., et al., 1991, MNRAS, 250, 24 